\title{
PAPER Re-Scheduling of Unit Commitment Based on Customers' Fuzzy Requirements for Power Reliability
}

\author{
Bo WANG $^{\dagger \mathrm{a})}$, You $\mathrm{LI}^{\dagger \mathrm{b})}$, Nonmembers, and Junzo WATADA ${ }^{\dagger \mathrm{c})}$, Member
}

\begin{abstract}
SUMMARY The development of the electricity market enables us to provide electricity of varied quality and price in order to fulfill power consumers' needs. Such customers choices should influence the process of adjusting power generation and spinning reserve, and, as a result, change the structure of a unit commitment optimization problem (UCP). To build a unit commitment model that considers customer choices, we employ fuzzy variables in this study to better characterize customer requirements and forecasted future power loads. To measure system reliability and determine the schedule of real power generation and spinning reserve, fuzzy Value-at-Risk (VaR) is utilized in building the model, which evaluates the peak values of power demands under given confidence levels. Based on the information obtained using fuzzy VaR, we proposed a heuristic algorithm called local convergence-averse binary particle swarm optimization (LCA-PSO) to solve the UCP. The proposed model and algorithm are used to analyze several test systems. Comparisons between the proposed algorithm and the conventional approaches show that the LCA-PSO performs better in finding the optimal solutions.

key words: customer requirements, power supply reliability, fuzzy set theory, fuzzy value-at-risk, particle swarm optimization algorithm, test systems
\end{abstract}

\section{Introduction}

Most previous studies have evaluated power loads forecasted using sufficient historical data. Then, using load prediction, grid corporations assign power generation tasks to each power plant without considering the possibility that power consumers can select different power supplies. The recent expansion of the electricity market enables consumers to purchase electricity of different quality and price according to their own requirements. Recently, Bilinton et al. [1], Niioka et al. [2] and Bilinton and Zhang [3] investigated consumers' preferences and requirements in the construction of their models.

Nevertheless, the current studies have three primary disadvantages. First, there are many factors that must be simulated or determined in order to find which factors will affect the real power loads. For example, weather, temperature and unexpected emergencies influence power consumption. As a result, power systems suffer from the uncertainty associated with the fact that the real power demands always deviate from forecasts [4]. Consequently, the use of

Manuscript received December 6, 2010.

Manuscript revised April 3, 2011.

The author are with the Department of Production, Graduate School of IPS, Waseda University, Kitakyushu-shi, 808-0135, Japan.

a) E-mail: bwangips@gmail.com

b) E-mail: rubyli@toki.waseda.jp

c)E-mail: junzow@ osb.att.ne.jp

DOI: 10.1587/transinf.E94.D.1378 historical information alone cannot predict real future demands. Secondly, in conventional models, the customer requirements for power supply reliability are expressed in real values such as $89.4 \%, 92.5 \%$ and $96.7 \%$, but generally speaking, most power consumers have only intuitive opinions on the required supply reliability. Real values cannot effectively describe imprecise and vague customer requirements. Thirdly, the existing studies assign a single value to the consumers' requirements during different periods of the day. In actuality, the power consumers always have different requirements for different periods. With respect to factories' electricity use, for example, for normal and efficient operation, the power supply reliability during working hours should always be higher than during non-working hours.

In this study, the conventional models are improved to mitigate the above shortcomings. The experts' opinions from related fields are taken into consideration to better describe the future power loads. To handle the uncertainty that arises from the use of historical data and expert opinions, it is better to treat the forecasted power loads as variables with imprecise distributions, i.e., fuzzy variables. The power supply reliability is described in fuzzy terms, as " $94 \%$ is preferred, but it must be higher than $88 \%$ ", or "the reliability is about $85 \%$ to $90 \%$ ". Such expressions are more acceptable for the power consumers when choosing their electricity supplies. Moreover, in this study, the customers are able to buy electricity of varied reliability for each time-period.

Employing fuzzy set theory, we take the forecasted loads and customers' reliability as fuzzy variables; the daily power demand is divided into 24 time-periods for each day (one hour per unit time). To deal with the fuzzy information, the fuzzy Value-at-Risk (VaR) is used to measure the reliability and determine the power demand and spinning reserve. After establishing the power generation and reserve, we must address a unit commitment problem (UCP) which is commonly a complex, nonlinear, mixed-integer optimization problem [4]-[6]. The conventional objective function is updated here by considering the spinning reserve costs in a fuzzy environment. To handle this UCP, we propose a local convergence-averse binary particle swarm optimization (LCA-PSO), which outperforms existing algorithms.

The remainder of this paper is organized as follows: in Sect. 2, we briefly review previous fuzzy power system problems and introduce some basic background regarding fuzzy variables and fuzzy VaR; Sect. 3 illustrates the proposed model; then, Sect. 4 provides the LCA-PSO as the solution; the model and algorithm are exemplified by some 
test systems in Sect. 5; finally, Sect. 6 summarizes the conclusions.

\section{Preliminaries}

Fuzzy set theory is a fundamental mathematical system that describes uncertainty, and various researchers have applied it to solve power system problems. To minimize real power losses and improve the voltage profile of a given system, Rahman [7] mathematically formulated an optimal reactive power control problem in which the objective function and constraints are expressed by fuzzy sets when modeled. In Khare's paper [8], fuzzy set theory was used to capture the subjective preferences of human operators in order to model their decision-making and generate a prioritized list of emergency problems that is more acceptable to the operator. Using a multi-objective fuzzy linear programming technique, Abou El-Ela [9] proposed a multi-stage procedure that lessens the influence of different emergency conditions. The results of their experiments showed that the proposed fuzzy procedure can reduce the influence of different emergency conditions.

Before discussing the fuzzy VaR, we preliminarily provide some basic concepts and features of fuzzy variables.

Suppose $\xi$ is a fuzzy variable whose membership function is $\mu_{\xi}$, and $r$ is a real number. Then, the possibility, necessity and credibility of event $\xi \geq r$ are expressed, respectively, as follows:

$$
\begin{aligned}
& \operatorname{Pos}\{\xi \geq r\}=\sup _{t \geq r} \mu_{\xi}(t), \\
& \operatorname{Nec}\{\xi \geq r\}=1-\sup _{t<r} \mu_{\xi}(t), \\
& \operatorname{Cr}\{\xi \geq r\}=\frac{1}{2}[\operatorname{Pos}\{\xi \geq r\}+\operatorname{Nec}\{\xi \geq r\}] .
\end{aligned}
$$

The credibility measure is formed on the basis of the possibility and necessity measures, and in the simplest case, it is taken as their average. The credibility measure is a selfdual set function [10], i.e. $\operatorname{Cr}\{\xi \geq r\}=1-\operatorname{Cr}\{\xi<r\}$. Equation (3) also results in the following:

$$
\operatorname{Cr}\{\xi \geq r\}=\frac{1}{2}\left[\sup _{t \geq r} \mu_{\xi}(t)+1-\sup _{t<r} \mu_{\xi}(t)\right] .
$$

For further information on fuzzy variables, one may refer to [10], [11] and [14].

According to [15], the VaR of an investment can be considered as the greatest value under a given confidence level. Following [12], if $\widetilde{L}_{t}$ denotes the fuzzy power demand of period $t$, in a fuzzy environment, the $\mathrm{VaR}$ of $L_{t}$ with a confidence of $(1-\beta)$ can be written as:

$$
\operatorname{VaR}_{1-\beta}=\sup \left\{\lambda \mid \operatorname{Cr}\left(\widetilde{L}_{t} \geq \lambda\right) \geq \beta\right\},
$$

where $\beta \in(0,1)$.

Equation (5) tells us that the greatest value of $\widetilde{L}_{t}$ under confidence level $(1-\beta)$ is the largest $\lambda$.

The following numerical example illustrates the calculation of the VaR.
Example 1. Supposing fuzzy variable $\widetilde{L}=F N(1.0,2.0)$, its membership function is normally distributed as $\mu_{\widetilde{L}}(r)=$ $\operatorname{Exp}\left\{-[(r-1.0) / 2]^{2}\right\}$, then let us calculate $\mathrm{VaR}_{0.9}$.

At first, we need to calculate the credibility function of $\widetilde{L} \geq r$. From Eq. (1), we can compute the possibility and the credibility of $\widetilde{L} \geq r$ in the following:

$$
\begin{aligned}
& \operatorname{Pos}\{\widetilde{L} \geq r\}= \begin{cases}1 & \mathrm{r} \leq 1.0 \\
\operatorname{Exp}\left\{-[(r-1.0) / 2]^{2}\right\}, & \mathrm{r}>1.0\end{cases} \\
& \operatorname{Nec}\{\widetilde{L} \geq r\}= \begin{cases}1-\operatorname{Exp}\left\{-[(r-1.0) / 2]^{2}\right\} & \mathrm{r} \leq 1.0 \\
0 & \mathrm{r}>1.0 .\end{cases}
\end{aligned}
$$

Hence, according to Eq. (3), the credibility of $\widetilde{L} \geq r$ is written as:

$$
\operatorname{Cr}\{\widetilde{L} \geq r\}= \begin{cases}1-\frac{1}{2} \operatorname{Exp}\left\{-[(r-1.0) / 2]^{2}\right\} & \mathrm{r} \leq 1.0 \\ \frac{1}{2} \operatorname{Exp}\left\{-[(r-1.0) / 2]^{2}\right\} & \mathrm{r}>1.0 .\end{cases}
$$

Therefore, making use of Eq. (5), we have,

$$
\operatorname{VaR}_{0.9}=\sup \{\lambda \mid \operatorname{Cr}(\widetilde{L} \geq \lambda) \geq 0.1\}=2.91 \text {. }
$$

Recently, the measure of Value-at-Risk has been applied to many engineering problems to build fuzzy optimization models, you may refer to [12], [13], [16]-[18], [20], [21], [23]-[25].

\section{Modeling}

The proposed model consists of two stages. In the first stage, customers choose power supplies for each period according to their requirements. Then, on the basis of the fuzzy power loads and customers' selections, the grid company forecasts the future demand and assigns the generation task to the power plants. In the second stage, with the purpose of providing sufficient electricity and reducing the generation cost, the UCP is solved by determining the power generation unit schedule. In the following, we explain the above two stages of the model in detail.

3.1 First Stage: Analyzing Forecasted Power Load and Customer Choices

The customer requirement for power reliability is expressed in natural language such as " $\mathrm{B} \%$ is preferred, but it must be higher than A\%" or "About A\% - B\%". Fuzzy variables are more suitable to denote such linguistic terms, whose membership function is shown in Fig. 1.

Here, $\mu(R)$ is the function that describes the reliability membership degree of the customers' requirement.

Based on the customers' requirements, the grid company provides $n$ alternative power supplies as shown in Fig. 2.

The forecasted power load at unit time $t$ is taken as a 


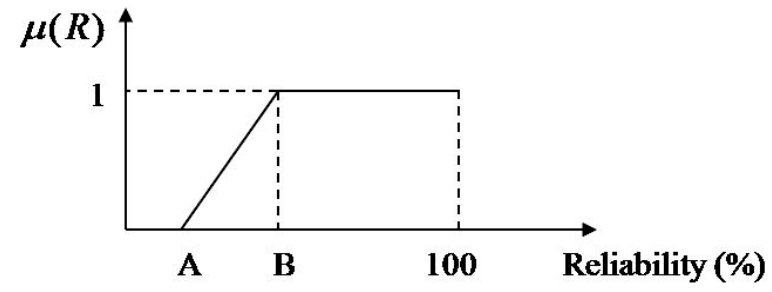

Fig. 1 Fuzzy membership function of customer requirements.

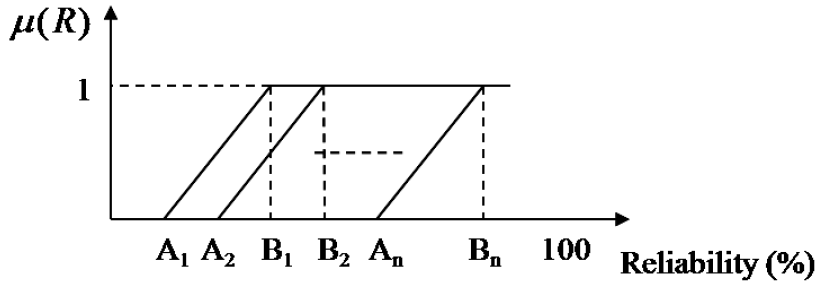

Fig. 2 Alternative power supplies.

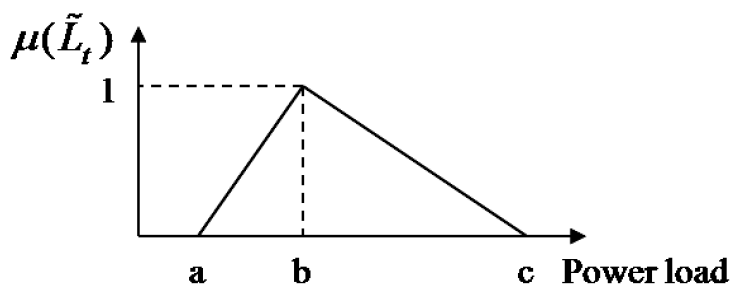

Fig. 3 Forecasted power load of unit time $t$.

fuzzy variable $\widetilde{L}_{t}$ as Fig. 3 shows.

The consumers choose the electricity while considering the supply reliability and price of power. Then, the grid company needs to determine the real generation and reserve in each period based on the fuzzy power load and consumers' choices.

\subsection{Second Stage: Re-Scheduling the UCP}

After pursuing the first stage, each power plant is assigned a certain amount of generation. It is their duty to provide sufficient real power and spinning reserve in each period. However, in a market environment, the profit is also important. Therefore, the majority of the previous studies summarized the UCP as minimizing the cost function without violating the constraints.

\subsubsection{The Conventional UCP}

A. Objective function

$$
\min F=\sum_{t=1}^{T} \sum_{i=1}^{N} u_{i}^{t} \cdot\left[f_{i}\left(P_{i}^{t}\right)+S C_{i}\left(1-u_{i}^{t-1}\right)\right] .
$$

Respectively, $F$ denotes the total cost of the power plant over the scheduling periods; $T$ and $N$ denote the number of considered system periods and the number of generation units, $P_{i}^{t}$ is the power generation of unit $i$ at time $t ; S C_{i}$

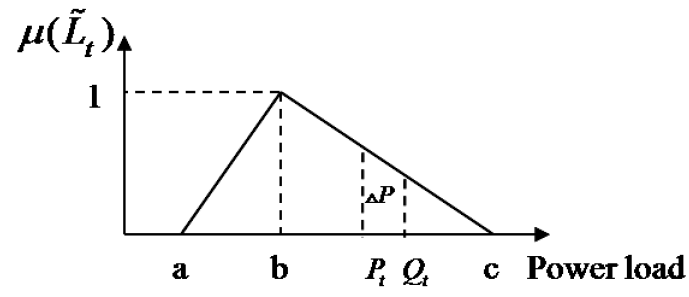

Fig. 4 The situation that spinning reserve is called.

is the start cost of unit $i$; $u_{i}^{t}$ denotes the status of unit $i$ at time $t . f_{i}\left(P_{i}^{t}\right)$ is the cost function of unit $i$ at time $t$, which is normally expressed by the following equation:

$$
f_{i}\left(P_{i}^{t}\right)=a_{i}+b_{i} P_{i}^{t}+c_{i}\left(P_{i}^{t}\right)^{2},
$$

where, $a_{i}, b_{i}$ and $c_{i}$ are the coefficients determined by the attributes of each generation unit.

\subsubsection{The Improved UCP}

A. Objective function

This study takes the spinning reserve cost into consideration. Then, in a fuzzy environment, the objective function is modified as follows:

$$
\begin{aligned}
\min F= & \sum_{t=1}^{T} \sum_{i=1}^{N} u_{i}^{t} \cdot\left\{\left[1-\operatorname{Pos}\left(Q_{t}>P_{t}\right)\right] f_{i}\left(P_{i}^{t}\right)\right. \\
& \left.+\operatorname{Pos}\left(Q_{t}>P_{t}\right) f_{i}\left(P_{i}^{t}+R_{i}^{t}\right)+S C_{i}\left(1-u_{i}^{t-1}\right)\right\} .
\end{aligned}
$$

$Q_{t}$ is the real load in period $t, \operatorname{Pos}\left(Q_{t} \geq P_{t}\right)$ is the possibility that the real demand is larger than the forecasted value $P_{t}, R_{i}^{t}$ is the spinning reserve of unit $i$ at time $t$, and $\sum_{i=1}^{N} R_{i}^{t}=R_{t}$, respectively.

\section{Remarks:}

$\square$ Supposing $\triangle P=Q_{t}-P_{t}$, if and only if $\Delta P>0$ (that is to say the real load is larger than the forecasted value as Fig. 4 shows), the reserve is considered to be called (even though the $\Delta P$ is sufficiently small).

$\square$ In an uncertain environment, the power loads are fuzzy variables, and subsequently, the possibility theory can be applied to measure the chance that the spinning reserve will be called:

$$
\operatorname{Pos}\left\{Q_{t}>P_{t}\right\}=\sup _{t>P_{t}} \mu_{\widetilde{L}_{t}}(t) .
$$

\section{B. Constraints}

The constraints are forwarded on the basis of system objective environmental demands. From the literature, they can be summarized as follows:

1). Power demand balance

$$
\sum_{i=1}^{N} P_{i}^{t} u_{i}^{t}=P_{t},
$$

where $P_{t}$ is the total power demand in period $t$. 
2). Spinning reserve balance

$$
\sum_{i=1}^{N} P_{\max } u_{i}^{t} \geq P_{t}+R_{t},
$$

where $R_{t}$ is the spinning reserve in period $t$.

$3)$. Interval of each generation unit

$$
P_{\min }^{i} \leq P_{i}^{t} \leq P_{\max }^{i},
$$

where $P_{\max }^{i}$ and $P_{\text {min }}^{i}$ are the maximum and minimum generation capacity of unit $i$, respectively.

4). Unit on/off limitations

Usually, the unit state should be kept on for certain hours before it can be changed.

$$
\left\{\begin{array}{l}
T_{i, \text { on }}^{t} \geq T_{i, \text { up }} \\
T_{i, \text { off }}^{t} \geq T_{i, \text { down }}
\end{array} .\right.
$$

where, respectively, $T_{i, o n}^{t}$ and $T_{i, o f f}^{t}$ are the time periods that unit $i$ has been turned on or off. $T_{i, u p}$ and $T_{i, \text { down }}$ are the minimum unit on time and maximum off time which are determined by the property of each unit.

5). Initial status of each unit

Before arranging the schedule, the unit initial status needs to be taken into consideration. That is, it is necessary to decide how many hours each unit has been committed or de-committed.

\section{The Solution}

In this section, we provide the solution for the proposed problem.

\subsection{The Strategy to Determine the Real Power Demands and Reserves}

In this study, based on the fuzzy Value-at-Risk, we provide the following strategy to decide the real power demand and reserve of each unit time. According to Sect. 2, the credibility function of the fuzzy power load $\widetilde{L}_{t}$ can be obtained as shown in Fig. 5.

Figure 2 shows the membership functions of the alternative power supplies. Supposedly, all the customers choose the power supply with the lowest reliability and price (the case of " $B_{1} \%$ is preferred, but it must be higher than $A_{1} \%$ ", shown in Fig. 2). That means, all of the customers' are satisfied with a supply reliability of $B_{1} \%$. Then, we calculate

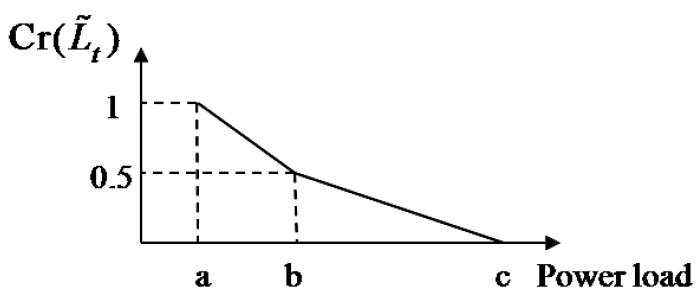

Fig.5 Credibility function of the fuzzy power load. the peak value of power loads under the supply reliability of $B_{1} \%$. In Eq. (5), set $1-\beta=B_{1} \%$, then under confidence level of $B_{1} \%$, the largest power demand of period $i$ is:

$$
\operatorname{VaR}_{B_{1} \%}=\sup \left\{\lambda_{i} \mid \operatorname{Cr}\left(\widetilde{L}_{i} \geq \lambda_{i}\right) \geq 1-B_{1} \%\right\},
$$

According to the computed result, if the generated electricity is no less than $\mathrm{VaR}_{B_{1 \%} \%}$, the power supply can be fully satisfied with reliability of $B_{1} \%$.

However, it is true that some customers want to buy power with higher reliability even though the price is higher. To provide sufficient power for all of the customers, a power reserve is required, and the reserve amount of period $i$ can be computed by the following Eq. (13):

$$
R_{i}=\sum_{j=2}^{n} \omega_{j} \cdot\left(\mathrm{VaR}_{B_{j} \%}-\mathrm{VaR}_{B_{1} \%}\right),
$$

where $R_{i}$ denotes the power reserve of period $i$, and $\omega_{j}$ is the coefficient that describes the proportion of customers who select a power supply with a reliability of $B_{j} \%$, respectively. The increasing expense of the spinning reserve is afforded by the customers with a higher expectation of electricity reliability. The higher the reliability they choose, the higher the power price will be.

This strategy is significant in two aspects: First, the basic requirement of power is probabilistically satisfied by the real generations. Second, the spinning reserve established by this approach can fulfill the power requirement in uncertainty environment under the given confidence level.

Repeating this strategy, we can determine the real values of 24-hour power demand and reserve.

\subsection{The Solution to UCP}

UCP has been solved using various kinds of heuristic algorithm, such as genetic algorithm (GA) [19], evolutionary programming (EP) [22] and lagrangian relaxation based PSO (LRPSO) [26]. Based on these methods, Yuan [6] proposed an improved binary PSO (IPSO) algorithm, which has proven to be more effective than other approaches.

In this research, based on original PSO [27], an LCAPSO is proposed to better solve the problem. The following is a preliminary explanation of the proposed method.

A. The fitness function

Considering the spinning reserve cost and fuzzy power load, the objective function is updated as Eq. (10), which is considered as the fitness function of LCA-PSO.

$\mathrm{B}$. The equations for the update of particle position and velocity

The equations proposed in classic binary PSO [28] are employed here. The particle position is updated by the following equations:

$$
\begin{aligned}
v_{i} & =w \cdot v_{i}+c_{1} \cdot \operatorname{rand}() \cdot\left(P_{\text {best }, i}-x_{i}\right)+c_{2} \cdot \operatorname{rand}() \cdot\left(G_{\text {best }}-x_{i}\right), \\
x_{i} & =x_{i}+v_{i},
\end{aligned}
$$

where, $v_{i}$ is the velocity of $i, \omega$ is the inertia weight, $c_{1}$ and $c_{2}$ 
are the learning rates generated in the interval $[0,4] \operatorname{rand}()$ is a random number distributed in the interval $[0,1] . P_{\text {best }, i}$ and $G_{b e s t}$ are the personal and global best among the iterations of particles, respectively. Subsequently, the position of particle $i$ is updated by Eq. (14).

Finally, the particles $0 / 1$ states are determined by a sigmoid function $S(x)$ :

$$
S(x)=\frac{1}{1+e^{-x_{i}}} .
$$

C. The local convergence of PSO

It is well known that the PSO algorithm can find the optimal solution in fewer iterations than other algorithms, but may suffer from the local convergence problem. In particular, when the selection problem contains a large number of objectives, this defect of the PSO becomes more obvious. To solve this problem, Yuan [6] performed 10 trials from different initial populations and selected the smallest cost as the result. Lee [29] proposed a mutation-based PSO (MPSO) where mutations occur with given possibility. In this paper, we design the LCA-PSO by employing the escape speed (ES) and velocity modification (VM).

Escape speed: If the optimal solution cannot be updated after a specified number of time iterations, it will be considered as having fallen into local convergence. Then, each particle speed will be modified by the escape speed. The value of ES should be determined by the experiments' performances: on one hand, if the escape speed is sufficiently large, all of the particles can jump out of the current loop and resume searching in different areas; on the other hand, if the escape speed is smaller, the new optimal solution can be obtained more quickly.

Velocity modification: After the above step, each particle's velocity has been modified to an opposite direction with a random value. This is inappropriate for the future search. Therefore, the VM is used to modify the velocity to a suitable value and in direction. In this case, a potential global optimal solution will be found.

We use the following two-dimensional Fig. 6 to approximate the LCA-PSO.

Based on the above analysis, we summarize the LCAPSO algorithm as follows:

[Step 1]. Initialize particles: Each particle contains $T \times$ $N$ generator states, as:

$$
x_{i}=\left[\begin{array}{c}
u_{1}^{1} u_{1}^{2} \cdots u_{1}^{T} \\
u_{2}^{1} u_{2}^{2} \cdots u_{2}^{T} \\
\cdots \cdots \cdots \\
u_{N}^{1} u_{N}^{2} \cdots \cdot u_{N}^{T}
\end{array}\right] .
$$

[Step 2]. Check the constraints of UCP, as mentioned in Sect. 3.2.2, part B.

[Step 3]. Solve the economic load dispatch (ELD) problem and update gray zone states for start-up cost. The concepts of ELD and gray zone have been well described in previous studies. One may refer to [6] and [30] for further information regarding these topics.

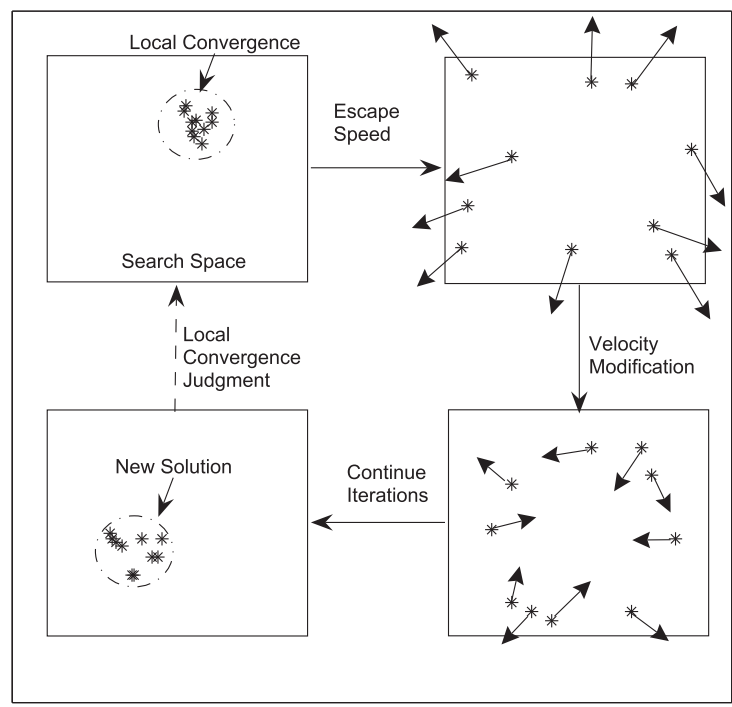

Fig. 6 Local convergence-averse BPSO.

[Step 4]. Initialize personal best and global best: The personal best and global best are determined by the fitness function (10).

[Step 5]. Particle iterations: The particle positions are updated by Eq. (14), and Step 2 is repeated to modify the unit states, then, new $P_{\text {best }}$ and $G_{\text {best }}$ are calculated.

[Step 6]. Solve the local convergence problem: If the global best cannot be improved in a given number of iterations, we consider the program to have fallen into the local convergence. Then, the aforementioned approach is used to cause the particles to jump out of current loop and to revise the particle's velocity.

[Step 7]. Following the completion of all iterations, the final global best is returned as the optimal result.

\section{Exemplification Using Test Systems}

\subsection{The Performances of the Proposed Model and LCA- PSO}

In this section, the proposed model and algorithm are exemplified using test systems. The 24-hour power loads are fuzzy variables evaluated from previous data and experts' opinions, as listed in Table 1. $(a, b, c)$ denotes the triangular fuzzy variable, and $\operatorname{FN}\left(a, \sigma^{2}\right)$ represents a normally distributed fuzzy variable. The power demand here are measured in megawatt (MW). Table 2 .

Three types of power supplies are available, as listed in

The power supply reliability is considered across the interval of [minimum reliability, maximum reliability].

Supposedly, from period 1 to 12 , each power supply scenario is selected by one-third of the customers; from period 12 to 24, one-fourth of the customers choose scenario I, whereas one-fourth choose scenario II, and the remaining half select scenario III.

We can determine the real power demand and reserve 
Table 124 hours forecasted fuzzy power demands.

\begin{tabular}{|c|c|c|c|}
\hline $\begin{array}{c}\text { Period } \\
1\end{array}$ & $\begin{array}{c}\text { Power Demands } \\
(650,700,750)\end{array}$ & $\begin{array}{c}\text { Period } \\
13\end{array}$ & $\begin{array}{l}\text { Power Demands } \\
(1350,1400,1450)\end{array}$ \\
\hline 2 & $(700,750,800)$ & 14 & $(1250,1300,1350)$ \\
\hline 3 & $(800,850,900)$ & 15 & $(1150,1200,1250)$ \\
\hline 4 & $(900,950,1000)$ & 16 & $(1000,1050,1100)$ \\
\hline 5 & $(950,1000,1050)$ & 17 & $\mathrm{FN}(1000,400)$ \\
\hline 6 & $(1050,1100,1150)$ & 18 & $\mathrm{FN}(1100,410)$ \\
\hline 7 & $(1100,1150,1200)$ & 19 & $\mathrm{FN}(1200,410)$ \\
\hline 8 & $\mathrm{FN}(1200,440)$ & 20 & $\mathrm{FN}(1400,430)$ \\
\hline 9 & $\mathrm{FN}(1300,420)$ & 21 & $\mathrm{FN}(1300,400)$ \\
\hline 10 & $\mathrm{FN}(1400,400)$ & 22 & $(1050,1100,1150)$ \\
\hline 11 & $\mathrm{FN}(1450,400)$ & 23 & $(850,900,950)$ \\
\hline 12 & $\mathrm{FN}(1500,390)$ & 24 & $(750,800,850)$ \\
\hline
\end{tabular}

Table 2 Available power supplies.

\begin{tabular}{ccc}
\hline Supply Scenario & Minimum Reliability & Maximum Reliability \\
1 & $70 \%$ & $80 \%$ \\
2 & $80 \%$ & $92 \%$ \\
3 & $90 \%$ & $99 \%$ \\
\hline
\end{tabular}

Table 324 hours evaluated power demands and reserves.

\begin{tabular}{cccccc}
\hline Period & Demands & Reserves & Period & Demands & Reserves \\
1 & 730.0 & 10.3 & 13 & 1430.0 & 12.5 \\
2 & 780.0 & 10.3 & 14 & 1330.0 & 12.5 \\
3 & 880.0 & 10.3 & 15 & 1230.0 & 12.5 \\
4 & 980.0 & 10.3 & 16 & 1080.0 & 12.5 \\
5 & 1030.0 & 10.3 & 17 & 1019.1 & 12.2 \\
6 & 1130.0 & 10.3 & 18 & 1119.4 & 12.3 \\
7 & 1180.0 & 10.3 & 19 & 1219.4 & 12.3 \\
8 & 1220.1 & 9.9 & 20 & 1419.4 & 12.7 \\
9 & 1319.6 & 9.7 & 21 & 1319.1 & 12.2 \\
10 & 1419.1 & 9.4 & 22 & 1130.0 & 12.5 \\
11 & 1469.1 & 9.4 & 23 & 930.0 & 12.5 \\
12 & 1518.9 & 9.3 & 24 & 830.0 & 12.5 \\
\hline
\end{tabular}

by using Eqs. (12) and (13). The results are shown in Table 3. Additionally, Eq. (11) computes the possibility that the spinning reserve might be called.

Based on the above analysis, the proposed LCA-PSO is evaluated through the use of different-sized test systems $(10,20,40,60,80$ and 100 units), which are expanded from a 10-unit based system [22]. All of the experiments are implemented on a Dell E8500 3.16 GHz CPU with $2.96 \mathrm{~GB}$ of RAM.

We first tested the LCA-PSO in different systems of various unit sizes. The parameters were selected as follows: 20 particles, 1000 iterations, $c_{1}=2.8, c_{2}=1.2, \omega=1$, a maximum speed of 4.0 and a minimum speed of -7.0 . For LCA-PSO, the given time iteration mentioned in Sect. 4 was set at 20, and this was used to evaluate whether the local convergence occurs.

Table 4 illustrates the performance of LCA-PSO by comparing the initial cost and the cost after 1000 iterations in different-sized systems. The savings are calculated as (InitialCost - FinalCost)/InitialCost $* 100 \%$.

From Table 4, it is obvious that, the LCA-PSO is an effective solution to the UCP, and this method could result in a cost-saving of more than $5.3 \%$.

In Tables 5 and 6, we show an example of initial and
Table 4 The performance of LCA-PSO

\begin{tabular}{cccc}
\hline Unit No. & Initial Cost & Final Cost & Savings \\
10 & 601,610 & 569,301 & $5.37 \%$ \\
20 & $1,207,189$ & $1,137,585$ & $5.77 \%$ \\
40 & $2,417,657$ & $2,274,514$ & $5.92 \%$ \\
60 & $3,622,180$ & $3,410,280$ & $5.85 \%$ \\
80 & $4,823,056$ & $4,561,093$ & $5.43 \%$ \\
100 & $6,027,820$ & $5,697,125$ & $5.49 \%$ \\
\hline
\end{tabular}

Table 5 The initial solution of LCA-PSO (cost: 601,610).

\begin{tabular}{|c|c|c|c|c|c|c|c|c|c|c|}
\hline $\begin{array}{ll} & \text { Unit No. }\end{array}$ & 0 & 1 & 2 & 3 & 4 & 5 & 6 & 7 & 8 & 9 \\
\hline 1 & 1 & 1 & 1 & 1 & 1 & 0 & 0 & 1 & 1 & 0 \\
\hline 2 & 1 & 1 & 1 & 1 & 1 & 0 & 0 & 0 & 0 & 1 \\
\hline 3 & 1 & 1 & 1 & 1 & 1 & 0 & 0 & 0 & 0 & 0 \\
\hline 4 & 1 & 1 & 1 & 1 & 1 & 1 & 1 & 1 & 1 & 1 \\
\hline 5 & 1 & 1 & 1 & 1 & 1 & 1 & 1 & 0 & 0 & 0 \\
\hline 6 & 1 & 1 & 1 & 1 & 1 & 1 & 1 & 0 & 0 & 0 \\
\hline 7 & 1 & 1 & 1 & 1 & 1 & 0 & 1 & 1 & 0 & 0 \\
\hline 8 & 1 & 1 & 1 & 1 & 1 & 0 & 1 & 0 & 0 & 1 \\
\hline 9 & 1 & 1 & 1 & 1 & 1 & 0 & 1 & 0 & 1 & 1 \\
\hline 10 & 1 & 1 & 1 & 1 & 1 & 1 & 1 & 0 & 0 & 0 \\
\hline 11 & 1 & 1 & 1 & 1 & 1 & 1 & 1 & 0 & 1 & 0 \\
\hline 12 & 1 & 1 & 1 & 1 & 1 & 1 & 1 & 0 & 0 & 1 \\
\hline 13 & 1 & 1 & 1 & 1 & 1 & 1 & 1 & 0 & 0 & 0 \\
\hline 14 & 1 & 1 & 1 & 1 & 1 & 1 & 1 & 1 & 1 & 0 \\
\hline 15 & 1 & 1 & 1 & 1 & 1 & 1 & 1 & 0 & 1 & 1 \\
\hline 16 & 1 & 1 & 1 & 1 & 1 & 1 & 1 & 1 & 1 & 0 \\
\hline 17 & 1 & 1 & 1 & 1 & 1 & 1 & 1 & 0 & 1 & 1 \\
\hline 18 & 1 & 1 & 1 & 1 & 1 & 1 & 1 & 0 & 0 & 1 \\
\hline 19 & 1 & 1 & 1 & 1 & 1 & 1 & 1 & 0 & 0 & 1 \\
\hline 20 & 1 & 1 & 1 & 1 & 1 & 1 & 1 & 0 & 0 & 1 \\
\hline 21 & 1 & 1 & 1 & 1 & 1 & 1 & 0 & 1 & 0 & 1 \\
\hline 22 & 1 & 1 & 1 & 1 & 1 & 1 & 0 & 0 & 0 & 0 \\
\hline 23 & 1 & 1 & 1 & 1 & 1 & 0 & 0 & 0 & 1 & 0 \\
\hline 24 & 1 & 1 & 1 & 1 & 1 & 0 & 0 & 0 & 0 & 0 \\
\hline
\end{tabular}

Table 6 The optimized solution of LCA-PSO (cost: 569,301).

\begin{tabular}{|c|c|c|c|c|c|c|c|c|c|c|}
\hline Hours $\quad$ Unit No. & 0 & 1 & 2 & 3 & 4 & 5 & 6 & 7 & 8 & 9 \\
\hline 1 & 1 & 1 & 0 & 0 & 1 & 0 & 0 & 0 & 0 & 0 \\
\hline 2 & 1 & 1 & 0 & 0 & 1 & 0 & 0 & 0 & 0 & 0 \\
\hline 3 & 1 & 1 & 0 & 0 & 1 & 0 & 0 & 0 & 0 & 0 \\
\hline 4 & 1 & 1 & 0 & 0 & 1 & 0 & 0 & 0 & 0 & 0 \\
\hline 5 & 1 & 1 & 0 & 0 & 1 & 0 & 0 & 0 & 0 & 0 \\
\hline 6 & 1 & 1 & 0 & 1 & 1 & 0 & 0 & 0 & 0 & 0 \\
\hline 7 & 1 & 1 & 0 & 1 & 1 & 0 & 0 & 0 & 0 & 0 \\
\hline 8 & 1 & 1 & 1 & 1 & 1 & 0 & 0 & 0 & 0 & 0 \\
\hline 9 & 1 & 1 & 1 & 1 & 1 & 0 & 0 & 0 & 0 & 0 \\
\hline 10 & 1 & 1 & 1 & 1 & 1 & 1 & 1 & 0 & 0 & 0 \\
\hline 11 & 1 & 1 & 1 & 1 & 1 & 1 & 1 & 0 & 0 & 0 \\
\hline 12 & 1 & 1 & 1 & 1 & 1 & 1 & 1 & 1 & 0 & 0 \\
\hline 13 & 1 & 1 & 1 & 1 & 1 & 1 & 1 & 0 & 0 & 0 \\
\hline 14 & 1 & 1 & 1 & 1 & 1 & 1 & 0 & 0 & 0 & 0 \\
\hline 15 & 1 & 1 & 1 & 1 & 1 & 0 & 0 & 0 & 0 & 0 \\
\hline 16 & 1 & 1 & 1 & 1 & 1 & 0 & 0 & 0 & 0 & 0 \\
\hline 17 & 1 & 1 & 1 & 1 & 1 & 0 & 0 & 0 & 0 & 0 \\
\hline 18 & 1 & 1 & 1 & 1 & 1 & 0 & 0 & 0 & 0 & 0 \\
\hline 19 & 1 & 1 & 1 & 1 & 1 & 0 & 0 & 0 & 0 & 0 \\
\hline 20 & 1 & 1 & 1 & 1 & 1 & 1 & 0 & 1 & 0 & 0 \\
\hline 21 & 1 & 1 & 1 & 1 & 1 & 1 & 0 & 0 & 0 & 0 \\
\hline 22 & 1 & 1 & 0 & 0 & 1 & 1 & 0 & 0 & 0 & 0 \\
\hline 23 & 1 & 1 & 0 & 0 & 1 & 0 & 0 & 0 & 0 & 0 \\
\hline 24 & 1 & 1 & 0 & 0 & 1 & 0 & 0 & 0 & 0 & 0 \\
\hline
\end{tabular}


Table 7 Optimal results of different algorithms.

\begin{tabular}{|c|c|c|c|}
\hline Unit No. Methods & LCA-PSO & MPSO & IPSO \\
\hline 10 & 569,301 & 575,782 & 600,892 \\
\hline 20 & $1,137,585$ & $1,152,383$ & $1,192,453$ \\
\hline 40 & $2,274,514$ & $2,316,431$ & $2,397,195$ \\
\hline 60 & $3,410,280$ & $3,496,880$ & $3,592,585$ \\
\hline 80 & $4,561,093$ & $4,688,605$ & $4,795,907$ \\
\hline 100 & $5,697,125$ & $5,859,228$ & $5,986,118$ \\
\hline
\end{tabular}

Table 8 Comparisons of CPU time (s).

\begin{tabular}{cc|ccc}
\hline \hline Unit No. & Methods & LCA-PSO & MPSO & IPSO \\
\hline 10 & & 18.22 & 15.34 & 16.08 \\
20 & 55.46 & 63.30 & 68.79 \\
40 & 270.67 & 312.22 & 282.66 \\
60 & 569.52 & 669.76 & 740.98 \\
80 & 1062.11 & 1280.91 & 1434.91 \\
100 & 1667.68 & 2119.79 & 2496.10 \\
\hline
\end{tabular}

final schedules as found by the LCA-PSO for the 10-unit test model. This illustrates the number of start-up and shutdown decisions made through the LCA-PSO optimization process.

\subsection{Comparisons between LCA-PSO and Other Improved PSO Algorithms}

In this subsection, we compare the LCA-PSO with IPSO (for the best result out of 10 trails) [6] and MPSO [29]. For MPSO, the mutation probability was 0.1 . After 1000 iterations, the total costs and CPU time of each algorithm are listed in Tables 7 and 8.

We found that, in most of the test systems, the LCAPSO was able to obtain better optimal solutions with less runtime cost than other approaches.

Nevertheless, compared with the CPU time used in prior work, more computation time is required to reach the final optimal solutions in this study. The reason for this could be that the objective function here is modified from Eqs. (8) to (10). Therefore, during each iteration, the cost was computed with respect to two options: 1 . The reserve was not called (as in the conventional UC models); 2 . The reserve was called. We must re-arrange different unit on/off schedules for each situation. Therefore, more time was required to obtain the optimal result.

To prove the effectiveness of LCA-PSO, we employed a 100-units test system, and Fig. 7 shows the performance of each method during the 1000 iterations.

Finally, in Fig. 7, it should be noted that, in the 100 units test system, the IPSO and MPSO are suffering from the local convergence problem. However, the proposed LCAPSO could overcome the local convergence effectively, the ES and VM were quite effective in solving such unit commitment optimization problem.

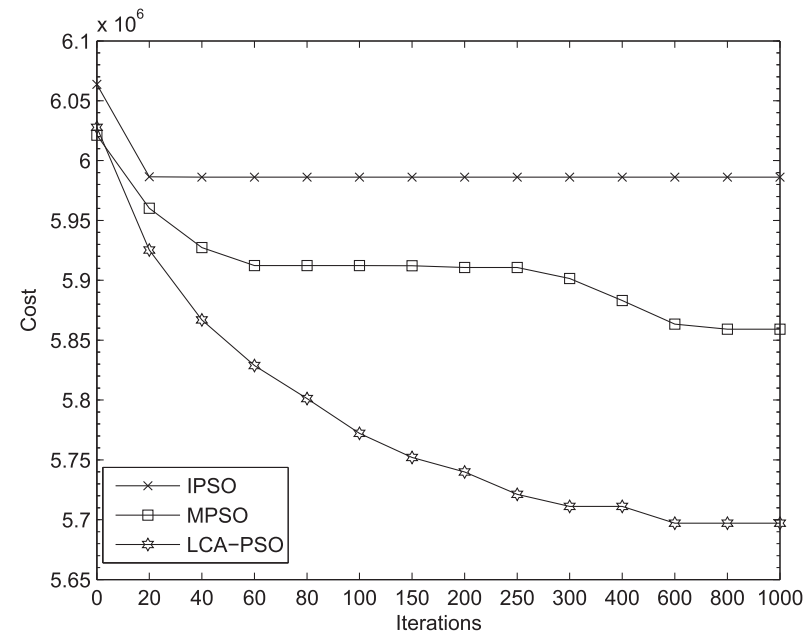

Fig. 7 Performances in a 100 unit test system.

\section{Conclusions}

In this study, we have proposed a fuzzy power system model that takes into consideration the customers' requirements. The fuzzy variable is used to more accurately describe the future power load and customers' requirements while the fuzzy Value-at-Risk is employed as a technique to evaluate the power demand and reserve for each period under a given confidence level. The conventional unit commitment model is improved by considering the possibility that the reserve is called. This model can minimize the system cost by considering various constraints and the customers' choices of power supply. To solve the problem, we proposed an LCAPSO as the solution that has been proved to be most useful and effective by the test systems. Furthermore, comparisons with existing algorithms show that the LCA-PSO has higher performance. In addition, the proposed model can be applied to various engineering reliability control problems.

\section{Acknowledgement}

This research was supported by Waseda University Global COE Program "International Research and Education Center for Ambient SOC" sponsored by MEXT, Japan.

\section{References}

[1] R. Billinton and W. Zhang, "Cost related reliability evaluation of bulk power systems," Int. J. Electrical Power and Systems, vol.23, no.4, pp.99-112, 2001.

[2] N. Okada and R. Yokoyama, "Evaluation and allocation of supply reliability cost in electricity market," Proc. IEEE PowerCon on 2000 International Conference Power System Technology, pp.703-707, 2000.

[3] R. Billinton, L. Cui, and Z. Pan, "Quantitative reliability considerations in the determination of the performance based rates and customer service disruption payments," Proc. IEE Generation, Transmission and Distribution, pp.640-644, 2002.

[4] P.A. Ruiz, C.R. Philbrick, K.W. Cheung, and P.W. Sauer, "Uncertainty management in the unit commitment problem," IEEE Trans. 
Power Syst., vol.24, no.2, pp.642-651, 2009.

[5] P. Attaviriyanupap, H. Kita, E. Tanaka, and J. Hasegawa, "A hybrid LR-EP for solving new profit-based UC problem under competitive environment," IEEE Trans. Power Syst., vol.18, no.1, pp.229-237, 2003.

[6] X. Yuan, H. Nie, A. Su, L. Wang, and Y. Yuan, "An improved binary particle swarm optimization for unit commitment problem," Expert Systems with Applications, vol.36, no.4, pp.8049-8055, 2009.

[7] H.A. Rahman and S.M. Shahidehpour, "A fuzzy based optimal reactive power control," IEEE Trans. Power Syst., vol.8, no.2, pp.662670, 1993.

[8] R. Khare and R.D. Christie, "Prioritizing emergency control problem with fuzzy set theory," IEEE Trans. Power Syst., vol.12, no.3, pp.1237-1242, 1997.

[9] A.A. Abou El-Ela, M.A. Bishr, S.M. Allam, and R.A. Ei-Sehiemy, "An emergency power system control based on the multi-stage fuzzy based procedure," Electric Power Systems Research, vol.77, no.5-6, pp.421-429, 2007.

[10] B. Liu and Y.K. Liu, "Expected value of fuzzy variable and fuzzy expected value models," IEEE Trans. Fuzzy Syst., vol.10, no.4, pp.445-450, 2002.

[11] S. Nahmias, "Fuzzy variable," Fuzzy Sets Syst., vol.1, no.2, pp.97101, 1978

[12] S. Wang, J. Watada, and W. Pedrycz, "Value-at-risk-based two-satge fuzzy facility location problems," IEEE Trans. Industrial Informatics, vol.5, no.4, pp.465-482, 2009.

[13] S. Wang and J. Watada, "Two-stage fuzzy stochastic programming with Value-at-Risk criteria," Applied Soft Computing, vol.11, no.1, pp.1044-1056, 2011.

[14] S. Wang, Y. Liu, and X. Dai, "On the continuity and absolute continuity of credibility functions," J. Uncertain System, vol.1, no.2, pp.185-200, 2007.

[15] D. Duffie and J. Pan, "An overview of value-at-risk," J. Derivatives, vol.4, no.3, pp.7-49, 1997.

[16] Y. Yoshida, "An estimation model of value-at-risk portfolio under uncertainty," Fuzzy Sets Syst., vol.160, no.22, pp.3250-3262, 2009.

[17] Z. Zmeskal, "Value at risk methodology under soft conditions approach (fuzzy-stochastic approach)," Eur. J. Oper. Res., vol.161, no.2, pp.337-347, 2005

[18] J. Peng and S. Li, "Analysis of different versions of the credibilistic value at risk," Proc. 6th International Conference on Fuzzy Systems and Knowledge Discovery, FSKD 2009, pp.96-100, 2009.

[19] S. Kazarlis and A. Bakirtzis, "A genetic algorithm solution to the unit commitment problem," IEEE Trans. Power Syst., vol.11, no.1, pp.83-92, 1996.

[20] B. Wang, Y. Li, and J. Watada, "Fuzzy power system reliability model based on Value-at-Risk," Proc. 14th International Conference on Knowledge-Based and Intelligent Information and Engineering Systems, pp.445-453, 2010.

[21] Z. Shang, H. Liu, X. Ma, and Y. Liu, "Fuzzy value-at-risk and fuzzy conditional value-at-risk: Two risk measures under fuzzy uncertainty," Proc. 2010 IEEE 2nd Symposium on Web Society, SWS 2010, pp.282-290, 2010.

[22] K. Juste, H. Kita, and E. Tanaka, "An evolutionary programming solution to the unit commitment problem," IEEE Trans. Power Syst., vol.14, no.4, pp.452-259, 1999.

[23] B. Wang, S. Wang, and J. Watada, "Fuzzy portfolio selection models with value-at-risk," IEEE Trans. Fuzzy Syst., vol.19, no.4, 2011, to be published.

[24] R.A. Jabr, "Robust self-scheduling under price uncertainty using conditional value-at-risk," IEEE Trans. Power Syst., vol.20, no.4, pp.1852-1858, 2005 .

[25] D. Xu and U. Kaymak, "Value-at-risk estimation by using probabilistic fuzzy systems," Proc. IEEE International Conference on Fuzzy Syst., pp.2109-2116, 2008.

[26] H. Balci and J. Valenzuela, "Scheduling electricity power generations using particle swarm optimization combined with the La- grangian relaxation method," Int. J. Applied Mathematics and Computer Science, vol.14, no.3, pp.411-421, 2004.

[27] J. Kennedy and R.C. Eberhaart, "Particle swarm optimization," Proc. 1995 IEEE International Conference on Neual Network, IV, pp.1942-1948, 1995.

[28] J. Kennedy and R.C. Eberhaart, "A Discrete Binary Version of the Particle Swarm Algorithm," Proc. IEEE International Conference on Systems, Man, and Cybernetics, pp.4104-4108, 1997.

[29] S. Lee, H. Park, and M. Jeon, "Binary particle swarm optimization with bit change mutation," IEICE Trans. Fundamentals, vol.E90-A, no.10, pp.2253-2256, Oct. 2007.

[30] X. Yuan, A. Su, Y. Yuan, H. Nie, and L. Wang, "An improved PSO for dynamic load dispatch of generators with valve-point effects," Energy, vol.34, no.1, pp.67-74, 2009.

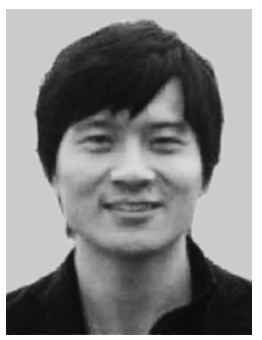

Bo Wang received the B.Sc. degree at the college of software engineering, Southeast University, Nanjing, China and the M.Sc. degree from Waseda University, Japan. He is currently working towards the Ph.D. degree at the Graduate School of Information, Production and Systems of Waseda University. $\mathrm{He}$ is a Research Assistant of the Global COE Program, Waseda University, Ministry of Education, Culture, Sports, Science and Technology, Japan. His research interests include soft computing, management engineering and optimal algorithms.

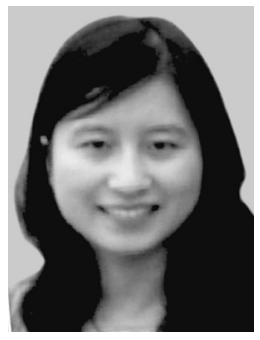

You $\mathbf{L i}$ received the B.Sc. degree from Nanjing Normal University Nanjing, China and the M.Sc. degree from Waseda University, Japan. She is currently working towards the $\mathrm{Ph} . \mathrm{D}$. degree at the Graduate School of Information, Production and Systems of Waseda University, Japan. Her research interests include management engineering and multi-objective optimization problems.

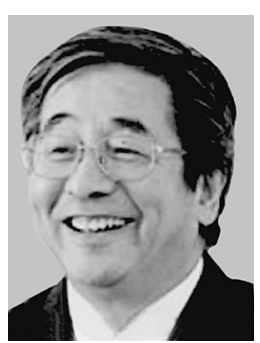

Junzo Watada received the B.Sc. and M.Sc. degrees in electrical engineering from Osaka City University, Osaka, Japan, and the Ph.D. degree from Osaka Prefecture University, Osaka. Currently, he is a Professor of Management Engineering, Knowledge Engineering and Soft Computing at the Graduate School of Information, Production Systems, Waseda University, Fukuoka, Japan. His professional interests include soft computing, tracking system, knowledge engineering, and management engineering. Prof. Watada is a recipient of the Henri Coanda Gold Medal Award from Invention in Romania in 2002. He is a Life Fellow of Japan Society for Fuzzy Theory and intelligent Informatics (SOFT) and Biomedical Fuzzy System Association (BMFSA). He is the Principal Editor, a Co-Editor, and an Associate Editor of various international journals, including the International Journal of Biomedical Soft Computing and Human Sciences, ICIC Express Letters, and the International Journal of Systems and Control Engineering, Fuzzy Optimization Decision Making. 